\title{
Risk factors for healing of duodenal ulcer under antacid treatment: do ulcer patients need individual treatment?
}

\author{
S MASSARRAT, H G MÜLLER AND P SCHMITZ-MOORMANN \\ From the Department of Gastroenterology and Metabolic Disease, Institute of Biomedical Statistics, and \\ Institute of Pathology, Philipps-University, Marburg, Federal Republic of Germany
}

SUMmARY In order to identify the risk factors affecting the healing of duodenal ulcer, a clinical trial with effective dose of antacid was carried out in 53 patients. Duration of ulcer history, number of relapses, duration of the last and present relapse, number, duration and severity of pain attacks in the present ulcer relapse, pain radiation to back, vomiting, appetite, smoking habit, intake of analgesics and previous haemorrhage were registered. Number of ulcers, ulcer depth, bublar narrowing, erosions, duodenitis at initial endoscopy and healing of ulcer were assessed by one endoscopist. Basic and peak acid output were measured. The extent of duodenitis on the site opposite the ulcer was determined by histological examination. Sixty per cent of the duodenal ulcers were healed after three weeks. By univariate analysis, the following factors affect the healing; pain radiation to back and pain duration during treatment $(p<0 \cdot 001)$, multiple or deep ulcers, narrowing of duodenal bulb $(\mathrm{p}<0 \cdot 01)$, number of pain attacks and poor appetite $(\mathrm{p}<0 \cdot 05)$. By the stepwise logistic regression model, the following factors were selected as predictors for healing of duodenal ulcer with $76 \%$ correct classification: pain radiation to back $(p=0 \cdot 002)$, deep ulcer $(\mathrm{p}=0 \cdot 013)$, multiple ulcers $(\mathrm{p}=0 \cdot 028)$. Number of cigarettes/day $(\mathrm{p}=0.007)$ and $\operatorname{male} \mathrm{sex}(\mathrm{p}=$ 0.036). By this model, the prediction of healing could be accurately assessed in $78 \%$ in a new sample. Individual treatment should be carried out on the basis of these factors.

In 1977 Peterson et al' observed the worsening effect of smoking on duodenal ulcer healing with antacid therapy, an affect which we confirmed using low dose antacids. ${ }^{23}$ We discussed the desirability of defining the severity of ulcer disease analogous of the way this is done for Crohn's disease. The clarification of severity of ulcer disease has been demanded in a recent publication on the principles related to the treatment of peptic ulcer. ${ }^{+}$In most recent reports of the last years there is almost general agreement that smoking delays healing of duodenal ulcer under various treatment regimes. ${ }^{1.35 .11}$ Nevertheless, there are many controversial reports on other factors affecting healing. ${ }^{12-15}$ Many of these reports are based on multicentre study where the quality of the collected

Address for correspondence: Prof Dr S Massarrat, Medizinisches Zentrum, Philipps-University, Baldingerstr, 355 Marburg. Federal Republic of Germany. Received for publication 7 August 1987 data is questionable because of the different views assessing the endoscopic findings and the severity of lesions. On the other hand, various treatment regimes were tested in most studies and it seems that the interest was focused on assessing the effectiveness of drugs but not primarily on elucidating the risk factors under one constant and effective regime.

In continuation of our efforts to find out the relevant factors affecting healing, ${ }^{3}$ we undertook a clinical short term trial in all cases under moderate and effective dose of antacid, where all clinical and endoscopic data were established by one gastroenterologist and endoscopist.

\section{Methods}

PATIENTS

During 2.5 years 76 German patients with duodenal ulcer were seen by one of the authors (SM). The 
patients were referred from general physicians and had no previous treatment with $\mathrm{H}_{2}$-receptorantagonists in the present ulcer relapse. The patients with ulcer complications were not entered in to the study. All endoscopies were carried out without sedation. The endoscopic findings were registered immediately after endoscopy. And on the same day either before or after endoscopy the patients were questioned by one person about their characteristics and clinical data. All patients were given $10 \mathrm{ml}$ antacid containing aluminium and magnesium hydroxide four times daily (Maalox 70), each dose having a neutralising capacity of $70 \mathrm{meq}$. HCI. All patients were recommended to register the intake of antacids and the appearance of pain daily on card and come to control endoscopic examination three weeks later. A gastric analysis was done either before or after the treatment in order to determine basic acid output (BAO) and peak acid output (PAO).

The following information was entered into the questionnaire: age, sex, height, weight, duration of ulcer symptoms, the approximate number of ulcer relapses during the length of disease, the length of the last ulcer recurrence, the length of the present ulcer relapse, approximate number of pain attacks during the present relapse, the severity of pain $($ mild $=1$, moderate $=2$, severe $=3)$, duration of pain attack $(15$ $\min =1,30 \mathrm{~min}=2,60 \mathrm{~min}=3,>60 \mathrm{~min}=4)$, radiation of pain to back, appetite (poor $=3$, moderate $=2$, good=1), vomiting (number/week), smoking habit, number of cigarettes smoked daily, duration of smoking habit (in years), the intake of analgesics (none $=1$, some times $=2$, regularly $=3$ ), previous haemorrhage or surgery. At the endoscopy the size of the largest ulcer niche in millimetres and the depth (flat $<3 \mathrm{~mm}$ or deep $\geqslant 3 \mathrm{~mm}$ in central area as two categories) were both assessed with the diameter of closed or opened tip of biopsy forceps. Furthermore, number of ulcers (single or multiple), grade of duodenal inflammation assessed only endoscopically (none $=0$, slight $=1$, moderate $=2$ and severe $=3$ ), the extent of bulbar deformity (none $=0$, partial wall deformity $=1$, moderate circular narrowing $=2$, severe circular narrowing $=3$, ulcer localized in the circumference of narrowed area of the bulb=4), and presence of antral erosions were registered. Biopsy specimens were taken from the opposite site of the ulcer niche in the duodenal bulb. The extent of duodenitis was classified histologically in five grades (from 0 to severe abnormality $=4$ ) according to the criteria of Whitehead and colleagues. ${ }^{\text {th }}$ On control examination the patients were asked about the number of days with regular pains and the regular intake of antacids by considering the daily cards. The healing of the ulcer was assessed and the ulcer was considered healed if the ulcer niche was completely or almost completely healed in the case of a nonmeasurable remnant.

STATISTICAL ANALYSIS

The characteristics of the two groups of successfully and unsuccessfully treated patients were compared by means of $\chi^{2}$ tests (discrete data), $t$ tests (continuous data) and Mann-Whitney U tests (rank data).

Twenty nine variables were chosen for these group comparisons. These univariate analyses were supplemented by a multivariate approach, which takes into account all variables simultaneously. We used stepwise logistic regression to find out which variables are jointly relevant for the prediction of ulcer healing. In the logistic regression model, the probability of healing success is modelled by the function

$$
\begin{aligned}
& \mathrm{P}=\mathrm{e}^{\mathrm{u}} /\left(1+\mathrm{e}^{\mathrm{u}}\right), \text { where } \mathrm{U}=\stackrel{\mathrm{k}}{\Sigma} \mathrm{ai} \times \mathrm{xi}+\mathrm{a}_{\mathrm{o}} \\
& \mathrm{i}=1
\end{aligned}
$$

$a_{i}, i=0, x_{1} \ldots x_{k} k$ denoting the $(k+1)$ parameters of the model and $x_{1} \ldots x_{k}$ denoting the independent variables (patient characteristics) used for the prediction.

The number of relevant variables $x_{1}$ as well as their parameters were determined by stepwise logistic regression (forward stepping), carried out by the program BMDPLR ${ }^{17}$ with the preassigned options. The resulting formula can be used to classify future patients into those with bad and others with good healing prognosis by defining a cutpoint on P (Probability), so that patients with a higher $P$ than the cutpoint are predicted to have a good healing prognosis.

The apparent correct classification rate, obtained by observing the proportion of the total sample which is classified correctly by the algorithm based on the same sample, is known to be a biased estimate of the correct classification rate and cannot therefore be used for the statistical validation of the algorithm. A method which avoids this problem is cross validation. This was done in the following way: Two thirds of the patients served as training sample from which the algorithm was computed and a random sample of about one third served as test sample for prediction. The proportion of correct classification in the test sample was assessed, where the patients in the test sample are different from those in the training sample from which the algorithm was constructed. Therefore this leads to a reasonable estimate of the correct classification rate. In order to improve this estimate further, the procedure of assessing correct classifications in a random test sample of about one third of the patients was repeated three times and the correct classification rate was estimated by a weighted average of the three observed correct classification 
rates. The reliability of the risk factors was shown by taking a new but small sample of nine patients, who were treated after the end of this trial or not included in the final evaluation, since the control endoscopy was not done by the endoscopist in charge of this study.

\section{Results}

Of 76 patients entered into the study, 23 dropped out: two patients took cimetidine because of sustained pain shortly after the beginning of treatment, two patients could not give exact history data, in one patient healing of ulcer could not be assessed by control because of bleeding during examination; in one patient control endoscopy was later than three weeks; six patients had control endoscopy by three other endoscopists, 11 did not come to control examination. Their initial data did not differ from that of the whole group. Therefore, 53 patients completed the study. In 32 of 53 the ulcer was healed (nine patients showed moderate healing, nine slight healing, in three patients the ulcer size was unchanged or worsened). In six patients gastric analysis could not be done. Complete data were available in 47 patients. Tables 1 and 2 show the clinical and endoscopic characteristics as well as others in healed and unhealed ulcer groups. The following factors have been found to be significant as unfavourable for healing: radiation of ulcer pain to back, the number of pain attacks during the present ulcer period before starting the treatment, the grade of appetite, the number of days with pain during the treatment. From the endoscopic view, deep and multiple ulcers as well as stenosis of duodenal bulb had a significant delaying effect on ulcer healing, while the size of the ulcer niche was of minor importance, just failing to reach statistical significance.

Although the number of smokers in relation to non-smokers in the non-healed group was almost twice as high as that in the healed group, this factor did not reach the limit of significance $(p=0 \cdot 32)$. The same was true for BAO, PAO and their weight corrected values in relation to ulcer healing. Sex, age,

Table 1 Characteristics of patients with healed and non-healed duodenal ulcer. Mean values (SD)

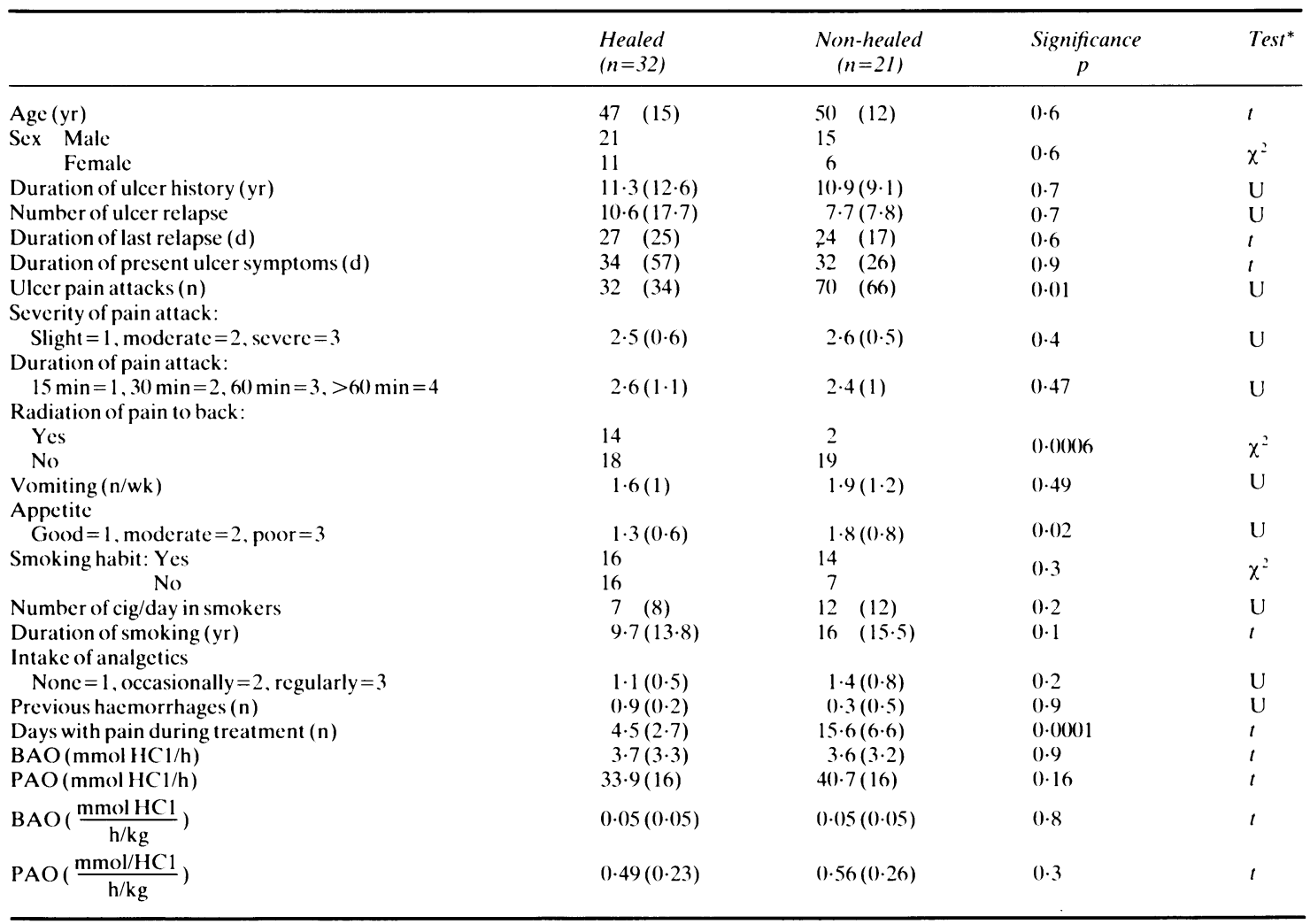

${ }^{*} t=$ t test, $\mathrm{U}=$ Mann-Whitney $\mathrm{U}$ test,$\chi^{2}=$ chi square test 
Table 2 Characteristics of endoscopic and histologic findings in healed and non-healed patients Mean (SD)

\begin{tabular}{|c|c|c|c|c|}
\hline & $\begin{array}{l}\text { Healed } \\
(n=32)\end{array}$ & $\begin{array}{c}\text { Non-healed } \\
\quad(n=21)\end{array}$ & $\begin{array}{c}\text { Significance } \\
p\end{array}$ & Test \\
\hline Ulcer size $(\mathrm{mm})$ & $6 \cdot 7(2 \cdot 8)$ & $8 \cdot 6(4 \cdot 1)$ & 0.055 & $t$ \\
\hline \multicolumn{5}{|l|}{ Ulcer depth: } \\
\hline Flat & 18 & 4 & \multirow{2}{*}{$0 \cdot 007$} & \multirow{2}{*}{$x^{2}$} \\
\hline Deep & 14 & 17 & & \\
\hline \multicolumn{5}{|l|}{ Ulcers $(n)$ : } \\
\hline Single & 31 & 14 & \multirow{2}{*}{0.002} & \multirow{2}{*}{$x^{2}$} \\
\hline Multiple & 1 & 7 & & \\
\hline \multicolumn{5}{|l|}{$\begin{array}{l}\text { Bulbar deformity: } 0=\text { none, } \text { slight }=1 \text {; } \\
\text { moderate narrowing }=2 ; \text { severe narrowing }=3 \text {, }\end{array}$} \\
\hline Antral erosions: Yes & 11 & 8 & \multirow{2}{*}{0.7} & \multirow[b]{2}{*}{$\chi^{2}$} \\
\hline None & 21 & 13 & & \\
\hline $\begin{array}{l}\text { Duodenitis (endoscopically): } \\
\text { none }=0 \text {; slight to severe }=1 \text { to } 3\end{array}$ & $2 \cdot 2(1 \cdot 1)$ & $2 \cdot 5(1 \cdot 1)$ & $0 \cdot 4$ & $\mathrm{U}$ \\
\hline $\begin{array}{l}\text { Duodenitis (histologically): } \\
\text { none }=0 \text {; slight to severe }=1 \text { to } 4\end{array}$ & $1 \cdot 4(1 \cdot 1)$ & $1 \cdot 5(1 \cdot 2)$ & 0.7 & $\mathrm{U}$ \\
\hline
\end{tabular}

endoscopically and histologically assessed duodenitis had, as individual factors, no effect on ulcer healing.

Logistic regression models are better suited to the analysis of mixtures of discrete and continuous data always encountered in clinical medicine rather than classical discriminant analysis which in a strict sense is interpretable only for continuous and normally distributed data. They are especially suited for the analysis of prognosis, as they yield a probability of success of treatment for each individual patient.

The logistic regression model, based on the total sample, chose sex (S) (female $=-1$, male $=+1$, $\mathrm{F}=4 \cdot 72, \mathrm{df}=1, \mathrm{p}=0 \cdot(036)$, radiation to the back (I) $($ no $=-1, \quad$ yes $=+1, \quad F=11 \cdot 06, \quad d f=1, \quad p=0 \cdot 002)$, number of cigarettes smoked per day (C) $(F=8 \cdot 02$, $\mathrm{df}=1, \mathrm{p}=0 \cdot 007)$, multiplicity of ulcer $(\mathrm{M})(\mathrm{no}=-1$, yes $=+1, F=5 \cdot 20, d f=1, p=0 \cdot(028)$, deep ulcer $(D)$ $(\mathrm{no}=-1$, yes $=+1, \quad \mathrm{~F}=6 \cdot 8, \mathrm{df}=1, \quad \mathrm{p}=0 \cdot 013)$, as predictors for the probability $(\mathrm{P})$ of ulcer healing. The fitted model is based on the following formula:

$$
\mathrm{U}(\text { total ulcer risk scores })=0.88-1 \cdot 14 \times \mathrm{S}-1.82 \times \mathrm{I}
$$$$
-0 \cdot 15 \times \mathrm{C}-2.37 \times \mathrm{M}-1.53 \times \mathrm{D} \text {, }
$$

where a worse prognosis was associated with male sex, radiation to the back, heavy smoking, multiple and deep ulcer. The worst values of the variables led to -5.98 in our sample. The corresponding probability of healing is $p<0 \cdot 0001$. The most favourable case led to a score of +5.98 and the corresponding probability of healing $\mathrm{P}$ is $100 \%(\mathrm{P}=1 \cdot 0$, see Figure $)$. The other factors were not selected as predictors.

The apparent correct classification rate for this model with a cutpoint at $\mathrm{P}=0.7$ - that is, classifying patients with higher $P$, or, equivalently with ulcer risk score $\mathrm{U}$ higher than $0 \cdot 85$, into the group with good prognosis, was $89.3 \%$ for the successful treatments and $90 \%$ for the unsuccessful ones. By the cross validation method described above, we estimated the correct classification rate to be $75 \cdot 1 \%$ (see below).

Observed classification for three random test samples (model computed without using the cases of the test samples)

$\begin{array}{lccc}\text { Samples } & 1 & 2 & 3 \\ \text { Correct } & 11 & 9 & 14 \\ \text { Wrong } & 3 & 5 & 3 \\ \% & 78 \cdot 6 & 64 \cdot 3 & 82 \cdot 4 \\ \text { mean }=75 \cdot 1 \% & & & \end{array}$

Each time a random test sample was chosen, the logistic regression model was based on the remaining data only. The selected variables were the same as those selected from the complete sample on all three occasions.

\section{Discussion}

The general policy for ulcer treatment is complete healing of the ulcer niche, which is associated with the disappearance of pain and late ulcer recurrence. Complete healing of the ulcer can be achieved in the majority of cases during four weeks effective treatment. However, most of the ulcers heal in less than four weeks with only a few taking longer. Therefore, it would be sound and logical to treat the patients for an adequate time if the probability of healing in the individual patient is predictable. As ulcer disease has multifactorial aetiology and geographic variation, the predictability of ulcer healing can only be achieved if the risk factors and their variability are known.

Many factors have been studied on ulcer healing: Among the characteristics of patients, there is age, ${ }^{612} 12^{27} \mathrm{sex}^{61 \times 27}$ duration of ulcer history ${ }^{12}$ and ulcer relapse, ${ }^{3}$ radiation of pain to back, ${ }^{\circ 1}{ }^{1 x}$ history 


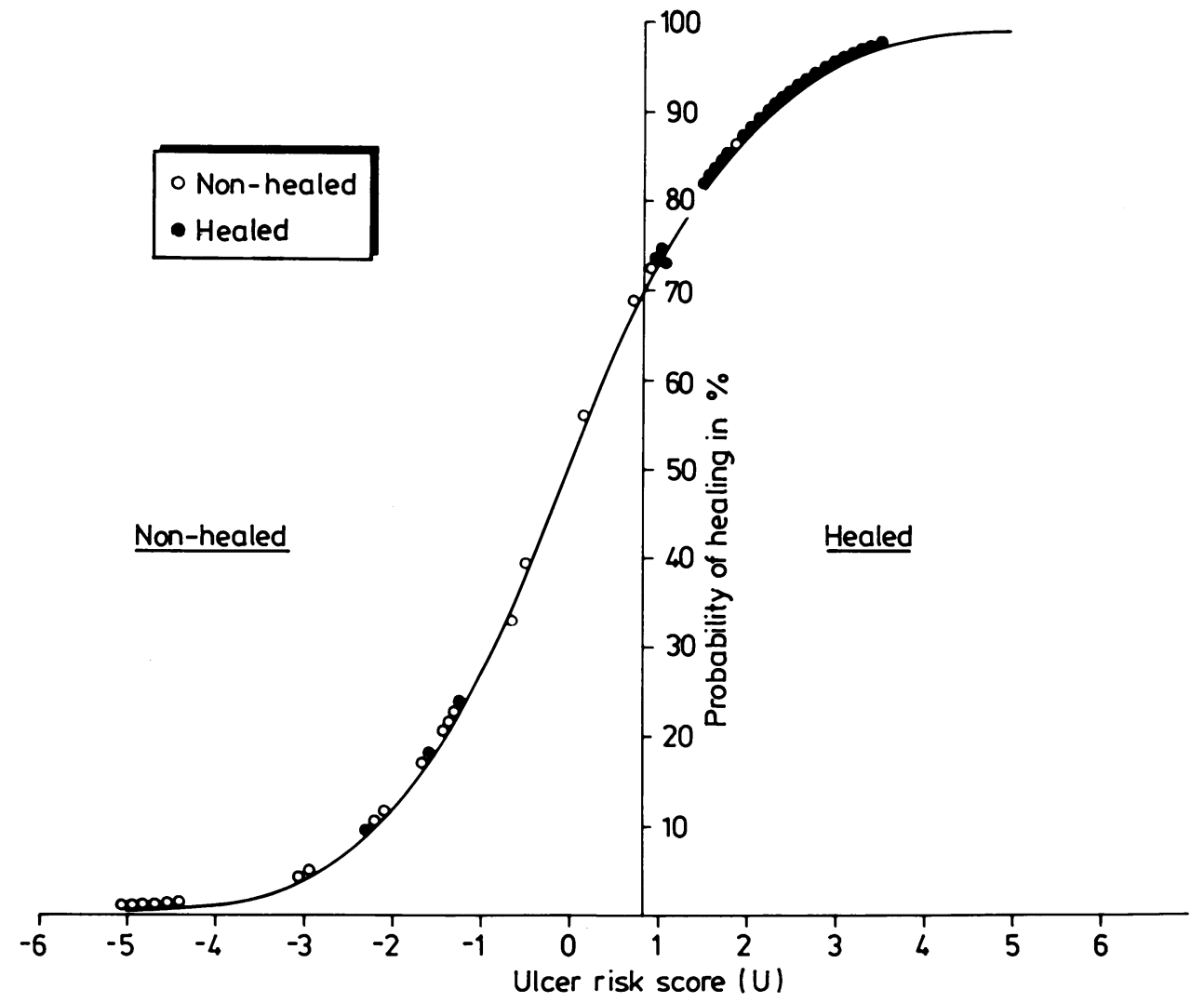

Fig. 1 Computation of the probability of healing (p) from the ulcer risk scores $U(U=0 \cdot 88-1 \cdot 14 \times S-1 \cdot 82 \times 1-0 \cdot 15 \times C$ $-2.37 \times M-1.53 \times D)$ according to the logistic regression model $\left(P=\frac{e^{u}}{I+e^{u}}\right)$.

of ulcer disease in close relatives, ${ }^{61112211}$ smoking habit, ${ }^{1.351527}$ alcohol intake, ${ }^{3111227}$ previous ulcer bleeding, ${ }^{12} \times 211$ and previous treatment with $\mathrm{H}_{2}$ receptor-antagonists. ${ }^{82}$ Among the endoscopic findings, there are ulcer diameter, ${ }^{13010112182112127}$ ulcer depth, ${ }^{18211}$ localisation of ulcer, ${ }^{21123}$ ulcer shape, ${ }^{1+22}$ macroscopically assessed duodenitis, ${ }^{12}$ duodenal deformity and stenosis ${ }^{3121 \times 211}$ and antral erosions. ${ }^{312}$ Further factors like BAO and $\mathrm{PAO}^{13 \times 91218212124}$ and serum pepsinogen $I^{1112+}$ have also been studied. Among these the importance of smoking, with few exceptions, ${ }^{12.1527}$ is recognised in many different countries. Its influence probably depends on further characteristics, however, such as the severity of smoking habit and the predisposition of patients.

There are many criticisms about most of these papers; in the cooperative studies the interobserver variations were not mentioned. Furthermore, the lack of multifactorial analysis by the statistical evaluation could mask the association of the relevant factors and disregard other important factors which have not been significant by using only univariate statistical analysis.

There are only five different author groups who presented such a multifactorial analysis. ${ }^{\circ \times 111182027}$ The relevant risk factors are different from one study to another, however, depending on the number and kind of factors considered as well as variable in the same group of patients during follow up treatment. ${ }^{21}$ In an earlier study, we found by stepwise regression analysis that stenosis of duodenal bulb, smoking habit and duration of present ulcer relapse of more than three weeks are unfavourable factors for healing during eight weeks treatment with a placebo like dose of antacids. ${ }^{3}$ The peak acid output, which was important as an individual factor, was associated with smoking. ${ }^{3526}$ In this paper we found under completely different conditions of treatment with effective dose of antacid and short treatment period of three weeks, that two of these three factors are of relevant importance; the stenosis of duodenal bulb by using statistical analysis of individual factors and smoking 
habit (the number of cigarettes smoked per day) by using logistic regression analysis. As an individual factor, the stenosis of the duodenal bulb has great importance; 19 of our cases had moderate to severe circular narrowing of the duodenal bulb. In 11 of these the ulcer niche was localised in the circumference of narrowed area. In 10 of these 11 cases, the ulcer niche did not heal at all. This important factor was removed in the logistic regression analysis, however, because of the association with other factors.

In disagreement with the assumption that endoscopically assessed duodenitis has a delaying effect on ulcer healing, ${ }^{12}$ we found that duodenitis either assessed by endoscopy or by careful histologic grading has no effect on healing.

The stepwise logistic regression procedure like multi-variate analysis techniques selects variables or factors that best represent a given series of patients with respect to healing and non-healing. It may, therefore, hide some related factors because these have been represented by the chosen factors but it may also reveal some less significant factors because together with the other selected factors these may better discriminate healing from non-healing. Thus, if a factor is not selected by the discriminant analysis, it does not necessarily mean that it is not important. Univariate analysis and multivariate analysis are, therefore, complementary to each other. This explains why apparently different factors are chosen by the two ways of analysis.

With the risk factors identified in this study, we could correctly predict ulcer healing in more than $75 \%$ of the cases. This prediction rate of healing was confirmed in a further small new sample of nine patients. In seven of these patients the prediction was correct $(78 \%$ correct classification rate). The reasons for the misclassification in a small number of patients may be statistical and medical. Medical reasons include the inadequate and incorrect answer of patients or incorrect assessment of endoscopic findings by the examiner. Statistical reasons include the importance of some factors initially not assessed as being a risk factor, attaining importance in the case of a lack of the other factors and random errors in the estimated model due to the sample of patients.

With the given risk factors, the calculation of the probability of the ulcer healing according to the above model is easily possible. The patients with calculated high ulcer score $U>0.85$ or equivalent estimated probability of healing $\mathrm{P}>0.7$ belong with about $80 \%$ confidence to the group of quick healers and need a symptomatic treatment during a few days as long as the pain is present. The patients with a calculated healing probability of $\mathrm{P}<0.3$ or ulcer risk score $U<0.85$ should be treated longer than four weeks and need not be checked by endoscopy earlier than 6 weeks. They are likely to require long term treatment. The patients with moderate calculated healing probability $(0 \cdot 7>\mathrm{P}>0.3$ or resp. medium ulcer risk score $-0 \cdot 85<\mathrm{U}<0 \cdot 85)$ should be treated in general for four weeks. These recommendations are valid for the conditions of our study. It is likely that under treatment with other drugs and long duration the factors differ. Once the factors under different treatments are known, it is hoped that the calculation of healing probabilities will be taken into consideration in general practice for reasons of economic treatment and in clinical studies for better assessment of the severity of the disease.

\section{References}

1 Peterson WL. Sturdevant RAL, Frankl HD, et al. Healing of duodenal ulcer with an antacid regimen. N Engl J Med 1977; 297: 341-5.

2 Massarrat S, Eisenmann A, Janzen R, Holle R. Prediction of a possibility of healing duodenal ulcer [Abstract]. Gastroenterology 1979; 76: 1195.

3 Massarrat S, Eisenmann A. Factors affecting the healing rate of duodenal and pyloric ulcers with low-dose antacid treatment. Gut 1981; 22: 97-102.

4 Chuong JJH, Spiro HM. Cimetidine and duodenal ulcer: an analysis of methodologic problems in randomized controlled trials. J Clin Gastroenterol 1982; 4: 311-20.

5 Lam SK, Lam KC, Lai CL, Yeung CK, Yam LYC, Wong WS. Treatment of duodenal ulcer with antacid and sulpiride. Gastroenterology 1979; 76: 315-22.

6 Sonnenberg A, Müller-Lissner SA, Vogel E. Prediction of duodenal ulcer healing and relapse. Gastroenterology 1981; 81: 1061-7.

7 Korman MG, Shaw RG, Hansky J, Schmidt GT, Stern AI. Influence of smoking on healing rate of duodenal ulcer in response to cimetidine or high-dose antacid. Gastroenterology 1981; 80: 1451-3.

8 Gugler R, Jensen JC, Rohner HG, Reimnitz P, Somogyi A. Factors predicting the treatment outcome of duodenal ulcer treatment with $\mathrm{H}_{2}$-receptor antagonists. Klin. Wochenschr 1982; 63: 1152-9.

9 Gugler R, Rohner HG, Kratochvil P, Brandstaetter G. "Effect of smoking on duodenal ulcer healing with cimetidine and oxmetidine". Gut 1982; 23: 866-71.

10) van Deventer GM, Schneidman D, Walsh JH. Sucralfate and cimetidine as single agents and in combination for treatment of active duodenal ulcers. A double blind, placebo-controlled trial. Am J Med 1985; 79: suppl 2c: 39-44.

11 Valnes K. Wetterhus S, Ellekjaer E. Trimipramine in the treatment of duodenal ulcer. A. multicenter open study. Scand J Gastroenterol 1980; 15: suppl 58: 65-70).

12 Bardhan KD. Refractory duodenal ulcer. Gut 1984; 25 : 711-17.

13 Barakat MW, Menon KN, Badawi AR. Cigarette smoking and duodenal ulcer healing, an endoscopic 
study of 197 patients. Digestion 1984; 29: 85-90.

14 Blum AL. Hat die Form des Ulcus duodeni prognostische Bedeutung bei der Cimetidin-therapie? Bericht über die Resultate einer Schweizer Multizenterstudie. Z Gastroenterol 1982; 26: 71-3.

15 Bianchi Porro G, Prada A, Petrillo M, Lazzaroni M. Gastric acid secretion, smoke and duodenal ulcer healing. [Correspondence]. Gastroenterology 1982; 82: 394-5.

16 Whitehead R, Roca M, Truelove SC. Antro-duodenitis in duodenal ulcers and non-ulcer dyspepsia. In: Wastell C, ed. Chronic duodenal ulcer. London: Butterworths, 1974: 17-23.

17 Dixon WJ. (ed) BMDP - Biomedical computer programs. Los Angeles: University of California Press, 1985.

18 Lam SK, Lai CL, Lee LNW, Fok KH, Ng MMT, Siu $\mathrm{KF}$. Factors influencing healing of duodenal ulcer. Control of nocturnal secretion by $\mathrm{H}_{2}$ blockade and characteristics of patients who failed to heal. Dig Dis Sci 1985: 30: 45-51.

19 Peden NR, Boyd EJS, Wormsley KG. Women and duodenal ulcer. $\mathrm{Br}$ Med J 1981; 282: 866.

20 Lam SK, Koo J. Accurate prediction of duodenal ulcer healing rate by discriminant analysis. Gastroenterology 1983; 85: 403-12.
21 Dive Ch, Fiasse R, Thome S, et al Rate of duodenal ulcer healing during treatment with omeprazole. A double-blind comparison of a daily dose of $30 \mathrm{mg}$ versus $60 \mathrm{mg}$. Scand J Gastroenterol 1986; 21: suppl: 175-6.

22 Kohli Y, Misaki F, Kawai K. Endoscopie follow-up observation of duodenal ulcer. Endoscopy 1972; 4: 202-8.

23 Lauritsen K, Bytzer P, Hansen J, Becker C. Comparison of ranitidine and high-dose antacid in the treatment of prepyloric of duodenal ulcer. Scand J Gastroenterol 1985; 20: $123-8$.

24 Quatrini M, Basilico G, Bianchi PA. Treatment of cimetidine-resistant chronic duodenal ulcers with ranitidine or cimetidine for eight weeks. Gut 1984; 25: 1113-7.

25 Parente F, Lazzaroni M, Sangaletti O, Baroni S, Bianchi Porro G. Cigarette smoking, gastric acid secretion, and serum pepsinogen I concentrations in duodenal ulcer patients. Gut 1985; 26: 1327-32.

26 Massarrat S, Enschai F, Pittner PM. Increased gastric secretory capacity in smokers without gastrointestinal lesions. Gut 1986; 27: 433-9.

27 Lux G, Hentschel H, Rohner HG, et al. Treatment of duodenal ulcer with low-dose antacids. Scand J Gastroenterol 1986; 21: 1063-8. 